\title{
Invited Response on: Dual-Plane Retro-Pectoral Versus Pre-pectoral DTI Breast Reconstruction: An Italian Multicenter Experience
}

\author{
Ugo Redi $^{1}\left(\mathbb{D} \cdot\right.$ Marco Marcasciano $^{2} \cdot$ Federico Lo Torto $^{1} \cdot$ Luca Patanè $^{1} \cdot$ \\ Diego Ribuffo ${ }^{1}$
}

Received: 23 December 2020/ Accepted: 25 December 2020

(C) Springer Science+Business Media, LLC, part of Springer Nature and International Society of Aesthetic Plastic Surgery 2021

Level of Evidence $V$ This journal requires that authors assign a level of evidence to each article. For a full description of these Evidence-Based Medicine ratings, please refer to the Table of Contents or the online Instructions to Authors www.springer.com/00266

First, we would like to thank the authors for their precious comments.

We truly appreciated the effort to offer an alternative and valuable point of view on such a trend topic, and we believe that scientific discussion can definitely help raise the bar in the complicated field of breast reconstruction.

We would like to take the chance to provide some kind of "historical" context to the study at issue and deepen the debate regarding some of the aspects highlighted in the discussion.

Both pre-pectoral and dual-plane direct to implant breast reconstructions have been shown to have benefits and drawbacks depending on each clinical scenario. For this very reason we do believe that selecting the most appropriate and targeted reconstructive technique for each individual patient is mandatory.

In this regard, the concept of this paper was born after several medical conventions, in which all of the authors reported a unanimous surgical trend, progressively shifting from dual-plane ADM-assisted breast reconstruction to a pure ADM pre-pectoral approach.

Ugo Redi

ugoredi88@gmail.com

1 Department of Surgery "Pietro Valdoni" Plastic Surgery Unit, Sapienza University of Rome, Rome, Italy

2 Department of Plastic and Reconstructive Surgery, San Gallicano Dermatologic Institute, IRCCS, Rome, Italy
All of us seemed to agree that this was probably the consequence of lower rates of complications and better aesthetic outcomes that were registered in each author's personal experience along different surgical departments across the State.

Besides sharing the same viewpoint, we decided to proceed further and design a retrospective study to investigate the scientific evidence elaborating a systematic analysis with consistent data, to verify and eventually offer support to what was still to be considered a personal surgical preference.

Concerning the infection risk, we agree with the authors that it might be favored by the presence of nonviable skin mastectomy flaps. In fact, this is one of the reasons why, as we thoroughly explain in the paper, in such cases we recommend a two-stage reconstruction with expanders.

Anyway, we believe that should there be any doubts concerning skin-edge viability, dual-plane reconstruction coverage of the prosthesis might be considered more robust only when the incision does not fall over the ADM.

The fact that the extra-dissection in a dual-plane reconstruction may be balanced out by the seroma-genic nature of the extra amount of ADM used in a pre-pectoral reconstruction was our starting point. Nonetheless the results we obtained and presented in the study confuted this theory. A very recent study [1] confirmed our findings, reporting that the elevation of the pectoralis muscle increased complications by threefold, after elimination of other confounding factors.

Obviously, other authors may achieve different outcomes.

When it comes to considering patient activity level in addition to flap thickness into reconstructive technique selection, we broadly agree. 
Unfortunately, in our experience, patients become extremely frustrated when animation deformity occurs as they often demand a corrective surgery. As you mentioned, studies reported that three-fourths [2, 3] of the patients were aware and bothered by the animation deformity, $51.9 \%$ of which reported that would have been interested in the avoidance of animation deformity with the initial surgical procedure [4].

We retain that quality of life is one of the main factors that are influenced by a specific breast reconstruction technique. This is why we try our best to avoid this complication independently of the athletic background of the patient.

Nipple areola reconstruction in pre-pectorally reconstructed patients still remains, in our opinion, individualized for each patient.

Harvesting local flaps is thoroughly dependent on subcutaneous tissue thickness in the area. Performing lipofilling 3 to 6 months after the first surgery usually permits to achieve adequate soft tissue coverage to the implant, also camouflaging one of the possible undesired outcomes such as the rippling deformity, and safely harvesting local flaps for nipple reconstruction. Finally, the areola may be reconstructed by tattooing the neighboring skin. Of course, this second operation (lipofilling) provides higher costs, with a view to the national healthcare and patient's psychological distress, but may be utilized to improve aesthetic appearance of the whole breast. When feasible, nipple reconstruction is always deferred at least 6 months after the first reconstructive step to ensure a stable underlying breast mound. Conversely, new approaching techniques comprehending NAC reconstruction by means of 3D tattooing are being performed [5]. Nipple reconstruction with local flaps is not suitable for every patient, and studies report heavy loss of nipple projection that may reduce patients' satisfaction over time [6], encouraging a surgeon and patient shift toward this new technique.

We concur with the authors that each surgeon should always have a clear idea of the cost efficiency ratio for each specific technique to make the best possible choice even in cost-effectiveness terms. Medical devices often increase surgical costs. Therefore, many practitioners across the world use synthetic meshes, less expensive devices that reportedly achieve good results. Others rather may prefer polyurethane implants for pre-pectoral reconstruction. These are all incredible tools that come at a price.

In this particular report, we use ADMs in both groups. Doing so, we did not notice a substantial difference in terms of costs between the two cohorts, despite the amount of ADM used. Nevertheless, their ability to reduce costs in terms of operating time and hospitalization encourages their use in the majority of international breast cancer centers [7]. Furthermore, the reduction of revision surgeries and complications of a total pre-pectoral reconstruction may enhance, as it is already happening in some centers, the spreading of this approach.

That said, we strongly agree that new papers are needed to better investigate the optimal option for breast reconstruction, considering both the patients' needs and the costs of the procedure. Nevertheless, we believe that in a multidisciplinary approach, surgeons are required to sew to measure a reconstructive option depending on the specific clinical scenario, avoiding to apply a particular technique to every patient, which may rather differ for general and local characteristics, tumor nature or oncological treatments.

\section{Compliance with Ethical Standards}

Conflict of interest The authors declare that they have no conflicts of interest.

Human and Animal Rights This article does not contain any studies with human participants or animals performed by any of the authors.

Informed Consent For this type of study informed consent is not required.

\section{References}

1. Gabriel A, Sigalove S, Storm-Dickerson TL et al (2020) DualPlane versus Prepectoral Breast Reconstruction in High-Body Mass Index Patients. Plast Reconstr Surg. 145(6):1357-1365. https://doi.org/10.1097/PRS.0000000000006840

2. Nigro LC, Blanchet NP (2017) Animation deformity in postmastectomy implant-based reconstruction. Plast Reconstr Surg Glob Open. 5:e1407

3. Spear SL, Schwartz J, Dayan JH et al (2009) Outcome assessment of breast distortion following submuscular breast augmentation. Aesthetic Plast Surg. 33:44-48

4. Casella D, Lo Torto F, Marcasciano M et al (2020) Breast Animation Deformity: A Retrospective Study on Long-Term and Patient-Reported Breast-Q Outcomes. Ann Plast Surg. https://doi. org/10.1097/SAP.0000000000002522

5. Hammond JB, Teven CM, Bernard RW et al (2020) 3D NippleAreolar Tattoo: It's Technique, Outcomes, and Utilization. Aesthetic Plast Surg. https://doi.org/10.1007/s00266-020-01967-w

6. Sisti A, Grimaldi L, Tassinari J et al (2016) Nipple-areola complex reconstruction techniques: A literature review. Eur J Surg Oncol. 42(4):441-465. https://doi.org/10.1016/j.ejso.2016.01.003

7. Glasberg SB (2017) The Economics of Prepectoral Breast Reconstruction. Plast Reconstr Surg. 140(6S):49S-52S. https:// doi.org/10.1097/PRS.0000000000004051

Publisher's Note Springer Nature remains neutral with regard to jurisdictional claims in published maps and institutional affiliations. 